\title{
Impact of ablation time on Cu oxide nanoparticle green synthesis via pulsed laser ablation in liquid media
}

\begin{abstract}
Large-scale commercial production of nanoparticles via efficient, economical, and environmentally friendly methods is a challenging endeavour. The laser ablation method being a green and potential route of nanoparticles synthesis can be exploited to achieve this end. In this work, we report the ablation of a copper target submerged in distilled water by pulsed Nd:YAG laser. The influence of ablation time on the structure and optical properties of grown copper oxide nanoparticles are studied. Such nanoparticle composition and structure is determined by X-ray diffraction (XRD), Fourier transform infrared, and Raman analyses. Results from transmission electron microscopy images established that synthesised nanoparticles are a spherical shape with average sizes of $24 i ̈ 37 \mathrm{~nm}$. Fluorescence spectra revealed the enhancement of nanoparticle concentration and reduction in the sizes with increasing ablation time, where the optimum ablation time is demonstrated to be 60 min. Photoluminescence spectra exhibited a prominent visible peak (green), which blueshifted from 542 to $537 \mathrm{~nm}$, confirming the shrinkage of copper oxide particle size at higher ablation time. The XRD pattern showed that the prepared nanoparticles possess a single phase of monocline cupric oxide nanostructure.
\end{abstract}

